\title{
Grüne Gentechnik oder Agrogentechnik
}

\author{
Werner Theobald $\cdot$ Friedhelm Taube
}

Erhalten: 15 Juli 2009/Akzeptiert: 17. Juli 2009/Online veröffentlicht: 4. August 2009

(C) Springer-Verlag 2009

Die Grüne Gentechnik oder Agrogentechnik ist die Anwendung genetischer Verfahren vornehmlich im Bereich der Züchtung von Pflanzen. Insbesondere bezeichnet der Begriff Verfahren zur Herstellung von gentechnisch veränderten Organismen (GVO), in deren Erbgut zusätzliche - in der Regel artfremde - Gene eingeschleust werden. Die Grüne Gentechnik ist somit ein Bestandteil der Grünen Biotechnologie.

\section{Bisherige Anwendungsbereiche}

Im Mittelpunkt der bisherigen Anwendungen der Grünen Gentechnik stehen weltweit vier Kulturpflanzen (in Klammern jeweils der Anteil an GVO, Stand 2008): Soja (72\%); Baumwolle (47\%); Mais (23\%) und Raps (21\%), wobei vor allem zwei Anwendungsbereiche relevant sind, für welche die Gentechnik in großem Umfang eingesetzt wird, nämlich die Herbizid- und Insektizidresistenz. Bei der Herbizidresistenz handelt es sich um eine Resistenz gegen so genannte Breitbandherbizide, die dadurch gekennzeichnet sind, dass alle anderen unerwünschten Pflanzenarten außer der GVO-Sorte abgetötet werden; dies ist insbesondere bei Soja und Mais von Bedeutung. Bei der Insektizidresistenz bewirken Gene des Bacillus thuringiensis (BT), die in den Zielorganismus eingeschleust wurden, dass diese Pflanzen eigenständig Gifte gegen bestimmte Schädlinge

W. Theobald $(\bowtie)$

Zentrum für Ethik der Universität Kiel,

Leibnizstr. 4, 24118 Kiel, Deutschland

E-Mail: werner.theobald@email.uni-kiel.de

F. Taube

Institut für Pflanzenbau und Pflanzenzüchtung,

Grünland und Futterbau/Ökologischer Landbau,

Christian-Albrechts-Universität Kiel,

Hermann-Rodewald-Str. 9, 24118 Kiel, Deutschland

E-Mail: ftaube@email.uni-kiel.de erzeugen. Dieser so genannte BT-Ansatz wird zurzeit vor allem bei Mais und Baumwolle verfolgt. Die BT-Toxine werden bereits seit Jahrzehnten direkt auch im biologischen Pflanzenschutz (z. B. im Ökologischen Landbau) eingesetzt.

\section{Forschungsbereiche}

In der Forschung stehen zurzeit andere Anwendungsgebiete neben den Herbizid- und Insektizidresistenzen im Mittelpunkt, namentlich die Trocken- und Salztoleranz bzw. die Veränderung von Wert gebenden Inhaltsstoffen von Kulturpflanzen, wobei bei Letzterem die Konsumenten als Zielgruppe angesprochen werden. Zwar ist derzeit ein breites Spektrum von GVO-Ansätzen aus dem oben genannten Bereich in Bearbeitung, jedoch ist die Praxisreife dieser GVOs zumeist noch nicht erreicht. Selbst der immer wieder als Positivbeispiel für die Grüne Gentechnik angeführte so genannte „Golden Rice“, welcher deutlich mehr Beta-Carotin, eine Vorstufe des Vitamin A, produziert und dem ein Einsatzgebiet in Ländern mit Vitamin A-Mangelernährung zugeordnet wird, hat noch nicht das Stadium des kommerziellen Anbaus erreicht.

\section{Argumente für die Grüne Gentechnik}

Als Argumente für die Grüne Gentechnik werden folgende Potentiale angeführt: höhere Erträge, Verbesserung der Widerstandsfähigkeit von Kulturpflanzen gegen Schädlinge, Krankheiten und ungünstige Umweltbedingungen, Verbesserung der Wert gebenden Inhaltsstoffe von Kulturpflanzen, z.B. eine veränderte Fettsäurezusammensetzung von Ölpflanzen oder die Entfernung von Allergenen für gesündere Lebensmittel - alles Ansätze, die mit konventionellen Züchtungsmethoden allein nicht umzusetzen wären. 


\section{Kritik an der Grünen Gentechnik}

Die Kritik an der Grünen Gentechnik fokussiert mögliche Nebenwirkungen, die sowohl nicht bekannte Veränderungen im Primär- und Sekundärstoffwechsel der Pflanzen betreffen könnten, als auch mögliche Gefahren für die Ökosysteme. Insbesondere die Auskreuzung von Resistenzgenen (z.B. Herbizidresistenz) in Wildkräutern ist diesbezüglich dokumentiert. Einige Mitgliedstaaten der EU kritisieren deshalb die ,ökologische Risikoabschätzung“ des GVO-Anbaus als unzureichend.

Schließlich ist ein übergeordnetes gewichtiges Argument der Kritiker der Grünen Gentechnik der Verweis auf sozioökonomische Gefahren, insbesondere für die durch kleinbäuerliche Strukturen geprägten Länder Afrikas, Asiens und Lateinamerikas. Dort reicht die Kaufkraft der Landwirte bereits heute nicht aus, um einfache Betriebsmittel wie Dünger einzusetzen. Insofern wird befürchtet, dass mit der großflächigen Einführung von GVO-Pflanzen die Abhängigkeit dieser Landwirtschaftskulturen von wenigen multinationalen Konzernen zunehmen könnte. Die Argumentationslinie dieser Kritiker hebt darauf ab, dass nicht technologische Einzellösungen (GVO) anzustreben seien, die aus den Industrieländern importiert werden, sondern Armutsbekämpfung durch ,good government“ in Verbindung mit der Entwicklung von standörtlich angepassten ökologisch nachhaltigen Landnutzungssystemen im Vordergrund stehen müsse.

\section{Grüne Gentechnologie in Deutschland}

Vor diesem Hintergrund sind die Agrar- und Ernährungswissenschaften in den Industrienationen gefordert, Modelle zu entwickeln, die unter Einbeziehung ethischer Grundsätze eine holistische Bewertung dieser neuen Technologie in einem Systemansatz gewährleisten. Als Basis dazu dient der Diskurs über die Grüne Gentechnologie in Deutschland.

Die Grüne Gentechnik (GGT) ist in Deutschland eine gesellschaftlich stark umstrittene Technologie. Bedenken von Umweltschützern und Verbraucherängste stehen in Konflikt mit den Interessen von Landwirten und forschender sowie vermarktender Industrie. Die Politik, auf diese Situation reagierend, ist selbst gespalten. Einerseits trägt sie durch Anbauverbote (wie kürzlich der Genmais-Sorte MON 810) einer pessimistischen Einschätzung der GGT Rechnung, andererseits fordert sie eine Versachlichung der Diskussion - nicht zuletzt deshalb, weil die GGT eine wichtige Zukunftstechnologie sei. Man brauche, so Bundesforschungsministerin Annette Schavan, dringend eine gesamtgesellschaftliche Diskussion, die sich auf sachliche, rationale Argumente stützen könne.

Wie könnte eine solche Diskussion aussehen und von wem sollte sie geführt werden?
Vorgeschlagen wird ein „Runder Tisch“ von Experten und Politikern, um Sachlichkeit in die Diskussion zu bringen, über die GGT objektiv zu informieren und damit zur Aufklärung in der Bevölkerung beizutragen. Doch woran sollte er sich orientieren?

\section{Grüne Gentechnik - Ein Bewertungsmodell}

Es gibt bisher nur wenige Studien, die eine rationale, wissenschaftliche Bewertung der GGT vornehmen. Die einzige, welche dies im deutschsprachigen Raum detailliert und ausführlich versucht hat, stammt aus dem Jahr 2002 (Busch et al. 2002). Interdisziplinär besetzt, entwickelt sie ein Bewertungsmodell, das als allgemein nachvollziehbar (und damit ethisch begründet) gilt, das sowohl methodisch als auch systematisch durchstrukturiert ist (und damit seine Wissenschaftlichkeit unter Beweis stellt) und das dafür entworfen wurde, um auf konkrete Einzelfallprobleme (wie z.B. MON 810) anwendbar zu sein (was die Politik ja interessiert).

Da dieses Modell gewissermaßen „monolithisch“ dasteht und als Basis für eine Bewertung der GGT in einem Systemansatz dienen kann, haben wir es zum Gegenstand einer eigenen Untersuchung gemacht (Theobald 2009). Wir beschäftigen uns damit aus zwei unterschiedlichen Perspektiven, die in zwei getrennten wissenschaftlichen Beiträgen zusammengeführt werden: zum einen - grundlegend - aus der Perspektive des an Bewertungsfragen interessierten philosophischen Ethikers (W. Theobald) und zum anderen - anwendungsbezogen - aus der Perspektive des an gentechnischen Fragen interessierten Agrarwissenschaftlers (F. Taube).

Die Analyse richtet sich zunächst (Teil 1, Theobald 2009) auf den methodischen Aufbau des Modells; sie untersucht die Schlüssigkeit seiner Argumente und unterzieht die Lösung, die es für das Problem GGT vorschlägt, einer eingehenden Diskussion (Teil 2, Theobald und Taube, eingereicht). Ohne die Ergebnisse dieser Diskussion hier vorweg nehmen zu wollen, kann bereits dies angedeutet werden: eine systematische Bewertung der GGT erweist sich als vielschichtiger und komplexer, als gemeinhin angenommen wird, und es darf als fraglich gelten, ob eine Expertenlösung im Sinne eines „Runden Tischs“ von Wissenschaftlern und Politikern wirklich die Lösung des Problems bringen wird.

\section{Literatur}

Busch JR, Haniel A, Knoeppfler N, Wenzel G (2002) Grüne Gentechnik. Ein Bewertungsmodell. Herbert Utz Verlag, München

Theobald W (2009) Grüne Gentechnik - Kritik eines Bewertungsmodells, Teil 1: Bewertungsgrundlagen: Umweltwiss Schadst Forsch 21(5), DOI 10.1007/s12302-009-0076-y 